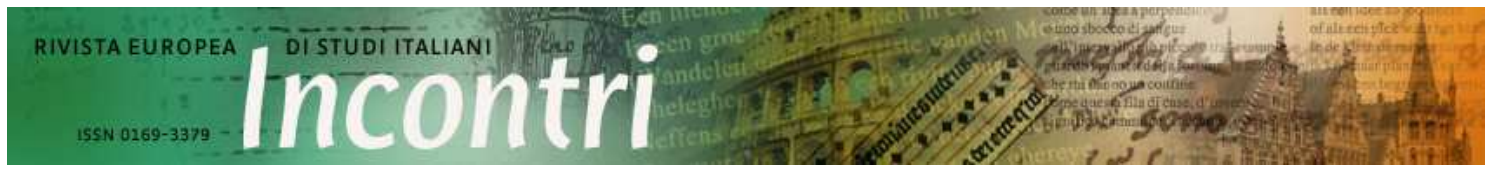

URN:NBN:NL:UI:10-1-101320 - Publisher: Igitur publishing

Content is licensed under a Creative Commons Attribution 3.0 License

Anno 26, 2011 / Fascicolo 1 - Website: www.rivista-incontri.nl

\title{
Prospettive diacroniche attraverso Roma antica
}

\author{
Recensione di: Amanda Claridge, Rome. An Oxford Archaeological \\ Guide, Oxford, Oxford University Press, 2010. ISBN: 9780199546831
}

\section{Alice Caltabiano}

'La Roma arcaica dei tempi di Romolo e la Roma sparita di fine Ottocento, sono entrambe risucchiate dal gorgo e dalla polvere della storia. Se dovessi indicare per la capitale un solo connotato punterei proprio su questo: la compresenza di tante città incastrate una dentro l'altra, sovrapposte in tre, quattro, cinque strati pronti a svelarsi appena si abbia voglia di guardare oltre la rumorosa corteccia del presente'. ${ }^{1}$

Con queste parole Corrado Augias offre, nel preambolo al suo libro, un'efficace visione prospettica di come si presenta oggi Roma agli occhi degli osservatori, di cosa significa per un visitatore moderno andare alla scoperta della Città antica.

Resta a tutt'oggi impresa assai difficile per i visitatori, per gli stessi abitanti, e talvolta anche per gli studiosi, conoscere a fondo l'infinito panorama che la città offre a chi si affaccia sulle sue rovine. La raccolta esaustiva delle manifestazioni artistiche, architettoniche, industriali, in una parola 'umane', è riunita nel bel volume dedicato a Roma delle Guide d'Italia del Touring Club dove vengono descritte con puntigliosa dovizia 'tre città' del tutto diverse tra loro. ${ }^{2}$ In un volume unico si ripercorrono tutte le epoche che attraverso la loro stratificazione hanno condotto alla realtà odierna: la Roma antica, la Roma cristiana e dei papi, la Roma capitale italiana. Nel corso di queste epoche tale è stato il susseguirsi della varietà di monumenti e opere d'arte, che il titolo dell'opera di Silvio Negro 'Roma, non basta una vita' è divenuta ormai una frase assai celebre. ${ }^{3}$

Nell'ambito delle guide dedicate esclusivamente alla città antica, ci si trova davanti una vasta offerta editoriale, di concezione e contenuti diversi. Per una visita esaustiva della città antica, si segnala in questa sede un'interessante versione rivolta

\footnotetext{
${ }^{1}$ C. Augias, I segreti di Roma. Storie, luoghi e personaggi di una capitale, Milano, Arnoldo Mondadori Editore S.p.A, 2005, p. 10.

${ }^{2}$ AA.VV. Roma, Guide d'Italia, Milano, Touring Club Italiano, 2002.

${ }^{3}$ S. Negro, Roma, non basta una vita, prefazione di E. Cecchi, Vicenza, Neri Pozza, 1962
} 
al pubblico anglofono, aggiornamento di una prima uscita del 1998, ricca di contenuti nuovi e abilmente strutturata.

A distanza di dodici anni, la collana Oxford Archaeological Guides, propone la nuova edizione della guida archeologica di Roma opera di Amanda Claridge, Professoressa di Archeologia Romana presso la Royal Holloway University of London e Assistant Director della British School at Rome dal 1980 al 1994. L'autrice è una specialista dell'arte romana, in particolare del commercio e della scultura dei marmi. Nel corso delle ricerche condotte a Roma, il suo interesse si è esteso anche all'architettura, all'urbanistica e alla topografia della capitale antica. La conoscenza delle opere è stata completata poi dalle ricerche degli studi antiquari tra il XVI e XVII secolo.

La nuova edizione, con prefazione di Barry Cunliffe, direttore della collana, Professore Emerito di Archeologia Europea presso la University of Oxford e membro della Ancient Monuments Board of English Heritage, è integrata rispetto all'antecedente versione con numerosi studi su aree archeologiche escluse dalla trattazione originale; inoltre essa è riorganizzata negli itinerari e aggiornata nelle informazioni di carattere pratico.

Uno degli aspetti più interessanti dell'opera risiede nella prima parte, dove l'autrice offre una sintetica ma accurata introduzione storica di Roma dalle origini fino alla tarda antichità, corredata da box esplicativi delle principali nozioni note al grande pubblico. Soprattutto, a margine della trattazione storica, mai superficiale, la Claridge propone un efficace e rapido rimando ai principali monumenti e musei che documentano le fasi storico-archeologiche della città (Roma monarchica, Roma repubblicana, e così via) così da offrire al visitatore un percorso di tipo cronologico. L'accostamento di opere contemporanee da parte dell'autrice è particolarmente apprezzabile dal momento che, così come si presenta oggi il patrimonio archeologico, l'impronta che più emerge è la magnificenza d'età imperiale. Meno decifrabili per i visitatori sono infatti i complessi più antichi, arcaici o repubblicani, rimaneggiati, ampliati o restaurati in età imperiale. Senza dimenticare che nel corso dei secoli, dal Medioevo fino all'Ottocento, le antichità vennero costantemente depredate, trasformate in cave di materiali da costruzione, andando così a rivivere in edifici e opere d'arte posteriori. La sincronia tra monumenti e reperti consente pertanto al visitatore di ricostruire la cultura materiale per singole facies, nel generale contesto evolutivo della città.

Sempre nella prima parte, al fine di esplicare al vasto pubblico le metodologie impiegate dagli studiosi per ricostruire vicende e topografia della città antica, vi sono $\mathrm{i}$ due sintetici capitoli sulle principali fonti documentarie e un glossario esaustivo per materiali da costruzione, tecniche edilizie, ordini e tipologie architettoniche, e principali opere pubbliche.

Nella descrizione dei monumenti la guida è organizzata secondo percorsi archeologicamente omogenei e attraverso tracciati significativi e chiarificatori delle diverse fasi; ciò dà modo al visitatore di scoprire porzioni ragionate della città antica, consentendogli di cogliere le numerose aree archeologiche nel corso di una giornata di cammino, arco temporale in armonia con i tempi dell'antichità. 
La visita è organizzata in modo radiale. Viene presentato il cuore politico e monumentale di Roma, con le sue tre aree distinte: il Foro Romano, il Palatino (con l'accesso tramite la via Sacra) e i Fori Imperiali. Nell'immediato circondario si articolano quindi i quartieri grossomodo omogenei tra loro: il Campo Marzio, il Campidoglio, la Valle del Circo Massimo e la Valle del Colosseo. Seguono poi i quartieri residenziali: l'Esquilino, il Celio con la porzione urbana della Via Appia e l'area del Laterano, il Viminale. Trattazione a parte è destinata all'antico quartiere portuale di Roma, l'area di Piramide-Testaccio, e infine alla sponda destra del Tevere, la moderna Trastevere. Tralasciando le aree di difficile comprensione perché prive di resti archeologici accessibili al pubblico, come l'Aventino, il Quirinale, e gran parte delle vie consolari, è infine presentato il parco archeologico della Via Appia Antica con gli imponenti edifici funerari e le residenze suburbane che lo caratterizzano; infine vengono descritte due piccole sezioni dedicate ad altre aree extraurbane, mentre un capitolo a parte è dedicato interamente alle catacombe. Questa lunga sezione destinata alla descrizione dei siti è corredata da un ampio apparato grafico cui rimandano i paragrafi descrittivi: le immagini, 200 circa, tutte in bianco e nero, sono tratte da pubblicazioni scientifiche che agevolano la lettura dei complessi archeologici, integrando alla descrizione mappe, piante e prospetti architettonici, ricostruzioni assonometriche e foto delle aree archeologiche e/o dei singoli monumenti.

L'apparato dedicato ai musei archeologici è integrato con la storia delle origini e con gli sviluppi delle collezioni, con la descrizione dei complessi architettonici che ospitano le raccolte, con il contenuto e l'esposizione dei reperti dei singoli poli museali, e infine con la descrizione delle opere di principale interesse storico, archeologico e artistico. In appendice sono consultabili una tavola cronologica, un elenco aggiornato di orari d'apertura e modalità d'accesso a monumenti, siti archeologici, musei, chiese e catacombe, e una vasta e aggiornata bibliografia ragionata per tematiche e per singoli siti. Conclude l'opera l'indice dei nomi.

Nel complesso, 540 pagine raccolte in un volume a copertina morbida, leggero e maneggevole, oltre che edizione tascabile, la guida di Amanda Claridge è un'esposizione dettagliata delle innumerevoli stratificazioni di Roma antica. Il testo offre gli elementi di conoscenza critica dei passaggi cruciali che hanno segnato il processo di sviluppo della città, dalle origini fino alla caduta dell'impero. Capace di rivolgersi ad un pubblico vasto e variegato, offrendo gli strumenti per comprendere tematiche e descrizioni storico-archeologiche trattate con accuratezza e metodologia scientifica, la guida è tuttavia lontana dagli stereotipi turistici, adeguata ad un pubblico di appassionati e, perché no, di specialisti. Nel visitare una città come Roma, unica al mondo per continuità di vita, le stratificazioni multiple che ne documentano la storia sono ben percepibili anche per occhi poco allenati alle testimonianze archeologiche. Quasi tre millenni di grandi progettazioni urbanistiche, opere d'arte pubbliche e private alternate ad incendi, saccheggi e bombardamenti si vedono, si sentono in ogni quartiere della città. Così come la trasformazione: residenze private divenute chiese cristiane; regge monumentali impiegate come fondazioni per complessi termali; porzioni di templi pagani impiegate per edificare 
mausolei pontifici, e così via. Tre città, si è detto in apertura; ebbene, pur andando alla scoperta di Roma antica, è inevitabile soffermarsi sulle città successive, poiché spesso sono i monumenti stessi a portare con sé le tracce del caotico susseguirsi di vicende che hanno segnato il destino di Roma. La convivenza e la contrapposizione di tutti questi elementi probabilmente è, e continuerà ad essere, l'elemento distintivo della città.

\section{Alice Caltabiano}

Dipartimento di Scienze storiche, archeologiche e antropologiche dell'antichità, Università degli studi di Roma 'La Sapienza', Via Palestro 63, 00185 Roma, Italia. alicecaltabiano@yahoo.it 